\title{
Risk Factors of Colorectal Stricture Associated with Developing High-Grade Dysplasia or Cancer in Ulcerative Colitis: A Multicenter Long-term Follow-up Study
}

\author{
Weimin $\mathrm{Xu}^{1}$, Wenjun Ding ${ }^{1}$, Yubei $\mathrm{Gu}^{2}$, Long $\mathrm{Cui}^{1}$, Jie Zhong ${ }^{2}$, and Peng $\mathrm{Du}^{1}$ \\ ${ }^{1}$ Department of Colorectal Surgery, Xinhua Hospital, Shanghai Jiao Tong University School of Medicine, and ${ }^{2}$ Department of Gastroenterology, \\ Rui Jin Hospital, Affiliated to Shanghai Jiao Tong University School of Medicine, Shanghai, China
}

See editorial on page 535.

Background/Aims: The risk factors of colorectal stricture associated with ulcerative colitis (UC) carcinogenesis in the long-term disease duration remain unclear. Methods: This study included all UC patients registered from a prospectively maintained database between June 1986 to July 2018 . The demographic data, clinical features, and outcomes in patients with dysplasia and stricture were assessed using univariable analysis and multivariate logistic regression models. Results: A total of 246 eligible patients were included in the analysis. The median follow-up time was 13.0 years (interquartile range [IQR], 9.0 to 16.0). There were 35 cases $(14.2 \%)$ of colorectal stricture. Patients with stricture had worse clinical outcomes. Stricture formation (odds ratio [OR], 9.350; 95\% confidence interval [Cl], 2.842 to 30.762), inflammatory polyps (OR, 5.464; 95\% $\mathrm{Cl}, 1.692$ to 17.638 ), disease duration of more than 10 years (OR, 3.223; $95 \% \mathrm{Cl}$, 1.040 to 9.985 ), and age $>40$ years at diagnosis (OR, 8.499; $95 \% \mathrm{Cl}, 1.903$ to 37.956 ) were significantly associated with high-grade dysplasia or colorectal cancer. In addition, disease duration of more than 5 years (OR, 3.211; 95\% Cl, 1.168 to 8.881), moderated anemia (OR, 3.373; 95\% Cl, 1.472 to 7.731 ), and primary sclerosing cholangitis (OR, 5,842 ; $95 \% \mathrm{Cl}, 1.395$ to 24.468 ) were contributing factors for the development of colorectal stricture. Conclusions: Colorectal stricture had the highest risk for malignant transformation. Earlier initiation of colonoscopic surveillance in UC patients with risk factors for stricture should be considered to prevent stricture formation and further malignant transformation. (Gut Liver 2020;14:601-610)

Key Words: Colitis, ulcerative; Stricture; Dysplasia; Colorectal neoplasms

\section{INTRODUCTION}

As a major form of inflammatory bowel disease (IBD), ulcerative colitis (UC) is characterized by a chronic course of recurrent relapse and remission, which markedly compromises the patient's quality of life. ${ }^{1}$ Despite advances in the treatment for UC, the therapeutic effects remain poor. ${ }^{2}$ Approximately 25\% to 30\% of UC patients ultimately need to undergo colectomy due to the ineffectiveness of drugs and disease complications such as colitisassociated neoplasia. ${ }^{3}$

Colorectal neoplasia is the most serious complication in UC patients, ${ }^{4-7}$ and there is a positive correlation between the cumulative risk of neoplasia and the duration of disease. ${ }^{8}$ Colorectal stricture may occur as a serious complication in UC, with an incidence ranging from $1.5 \%$ to $11 \% .^{9-12}$ Notably, a previous study has shown that colorectal stricture increased the risk of neoplasia in UC patients and caused disease complications that require colectomy. ${ }^{13}$ However, few studies have reported the risk factors of the stricture in the long-term disease duration.

This study aimed to assess the impact of stricture on clinical outcomes of UC to further confirm the severity of stricture. More impor-

\footnotetext{
Correspondence to: Peng $\mathrm{Du}^{\mathrm{a}}$ and Yubei $\mathrm{Gu}^{\mathrm{b}}$

${ }^{a}$ Department of Colorectal Surgery, Xinhua Hospital,1665 Kongjiang Road, Shanghai 200092, China

Tel: +86-21-25077858, Fax: +86-021-65030840, E-mail: dupeng@xinhuamed.com.cn

${ }^{b}$ Department of Gastroenterology, Rui Jin Hospital, Affiliated to Shanghai Jiao Tong University School of Medicine, 197 Rui Jin Er Road, Shanghai 200025, China

Tel: +86-021-64370045, Fax: +86-021-65030840, E-mail: gyb11809@rjh.com.cn

Received on July 5, 2019. Revised on September 11, 2019. Accepted on October 4, 2019. Published online December 11, 2019.

pISSN 1976-2283 eISSN 2005-1212 https://doi.org/10.5009/gnl19229

Weimin Xu and Wenjun Ding contributed equally to this work as first authors.

@ This is an Open Access article distributed under the terms of the Creative Commons Attribution Non-Commercial License (http://creativecommons.org/licenses/by-nc/4.0) which permits unrestricted non-commercial use, distribution, and reproduction in any medium, provided the original work is properly cited.
} 
tantly, we first discovered the risk factors of colorectal stricture in UC patients, which could provide some implications for clinical practice to identify the potential risk of stricture, earlier conduct endoscopic surveillance to reduce the incidence of stricture or neoplasia.

\section{MATERIALS AND METHODS}

\section{Study population}

All consecutive UC patients who underwent long-term and regular medical treatment or surgery at Xinhua Hospital and Rui Jin Hospital, Shanghai Jiao Tong University School of Medicine (Shanghai, China) from June 1986 to July 2018 were identified from a prospectively maintained, institutional review boardapproved database (Chinese Database System for IBD). The surgery mentioned above refereed to colectomy. We included UC patients aged $\geq 18$ years and received a regular follow-up at our department. Meanwhile, those with previous colorectal cancer (CRC) history, familial adenomatous polyposis or indeterminate colitis, and lost to follow-up were excluded. The Ethics Committee of Xinhua Hospital approved this study (approval number: XHEC-D-2018-088). Written informed consent was obtained.

\section{Data collection and clinical evaluation}

The clinical profiles of UC patients were retrospectively collected from the hospital medical records. The following clinical data were collected: sex, age at diagnosis, relapse, gastrointestinal symptoms, weight loss, extraintestinal manifestation, comorbidity, disease extent, disease duration, history of surgery, familial history of IBD or CRC, use of steroids and immunomodulators, and laboratory results (hemoglobin and albumin). History of surgery referred to any surgery prior to treatment of UC. Disease extent was categorized according to the Montreal classification system, ${ }^{14}$ and the diagnosis of UC was based on clinical, radiological, endoscopic examination, and histologic findings. Comorbidity was defined as primary sclerosing cholangitis (PSC) before UC diagnosis. The complications in the present study refer to various clinical complications during hospitalization and malignant transformation in the long-term follow-up. Serious complications were defined as bleeding, intestinal obstruction, or perforation. Additionally, bleeding referred to patients having significant bleeding in the lower gastrointestinal, not just bloody stool or presented with significant signs of anemia. All complications were diagnosed based on clinical manifestations, laboratory results along with endoscopic and imaging findings. Moreover, the diagnosis of colonic stricture with fibrotic luminal narrowing was mainly determined by imaging procedures or colonoscopy occurring during the period of following-up in the present retrospective study. A stricture was defined as a fixed, localized colonic narrowing and an endoscope could not pass through. ${ }^{15}$ Radiographically identifiable strictures were also included. Obvious polypoid lesions producing narrowing of the lumen and transient stricture caused by inflammatory edema of the intestine were excluded. Colorectal structures were identified in 35 patients, initially diagnosed either by barium enema or computed tomography (five patients), colonoscopy (28 patients), or surgery (two patients).

\section{Statistical analysis}

SPSS version 19.0 software (IBM Corp., Armonk, NY, USA) was used for statistical analysis. The statistical methods were similar to those in our previous study. ${ }^{16}$ In brief, results are reported in numbers and percentages, mean and standard deviations (SD), or the median and interquartile range (IQR). Unpaired Student t-test analysis and chi-square or Fisher exact test were used for analysis, as appropriate. Multivariate logistic regression was further performed to evaluate the risk factors of high-grade dysplasia or CRC and colorectal stricture in UC disease duration, with adjustments for variables that may be significant prognostic factors according to the univariate analyses. Confidence intervals (CI) were set at 95\%. All statistical tests were two-sided, with a p-value of $<0.05$ considered statistically significant.

\section{RESULTS}

\section{Patient characteristics}

Of the 271 UC patients who underwent medical treatment or surgery in our institution, 25 were lost to follow-up, one of them had the familial adenomatous polyposis and another one had indeterminate colitis, leaving 246 UC patients included in the current analysis. The characteristics of the study population are shown in Table 1 . The men and women were 120 and 126, respectively. The median follow-up time was 13.0 years (IQR, 9.0 to 16.0 years) from June 1986 to July 2018 in the whole cohort. The median age at diagnosis was 42.0 years (IQR, 29.5 to 54.0 years), and the median disease duration was 6.0 years (IQR, 4.0 to 10.0 years). Among the 246 patients, six (2.4\%) had proctitis (E1), 124 (50.4\%) had left-sided colitis (E2), and 116 (47.2\%) had pancolitis (E3). Moreover, extraintestinal manifestation, comorbidity (PSC), and familial history of IBD or CRC were present in $11.0 \%, 4.1 \%$, and $2.8 \%$ of patients, respectively. In addition, $63.0 \%$ and $12.2 \%$ patients were previously treated with steroids and immunomodulators.

\section{Analysis of complications and outcomes in UC patients}

Table 2 shows the various complications noted during the investigation. A total of 95 patients (38.6\%) experienced complications that included colorectal stricture $(n=35,14.2 \%)$, gastrointestinal bleeding $(n=25,10.2 \%)$, intestinal obstruction $(n=21$, $8.5 \%)$, colon perforation $(n=6,2.4 \%)$, toxic megacolon $(n=2$, $0.8 \%)$, Crohn's disease (CD) $(n=2,0.8 \%)$, inflammatory polyps $(\mathrm{n}=57,23.2 \%)$, and abscess $(\mathrm{n}=3,1.2 \%)$. In addition, 19 patients (7.7\%) underwent malignant transformation; of them, eight patients (3.3\%) developed high-grade dysplasia and 11 (4.4\%) developed CRC. 


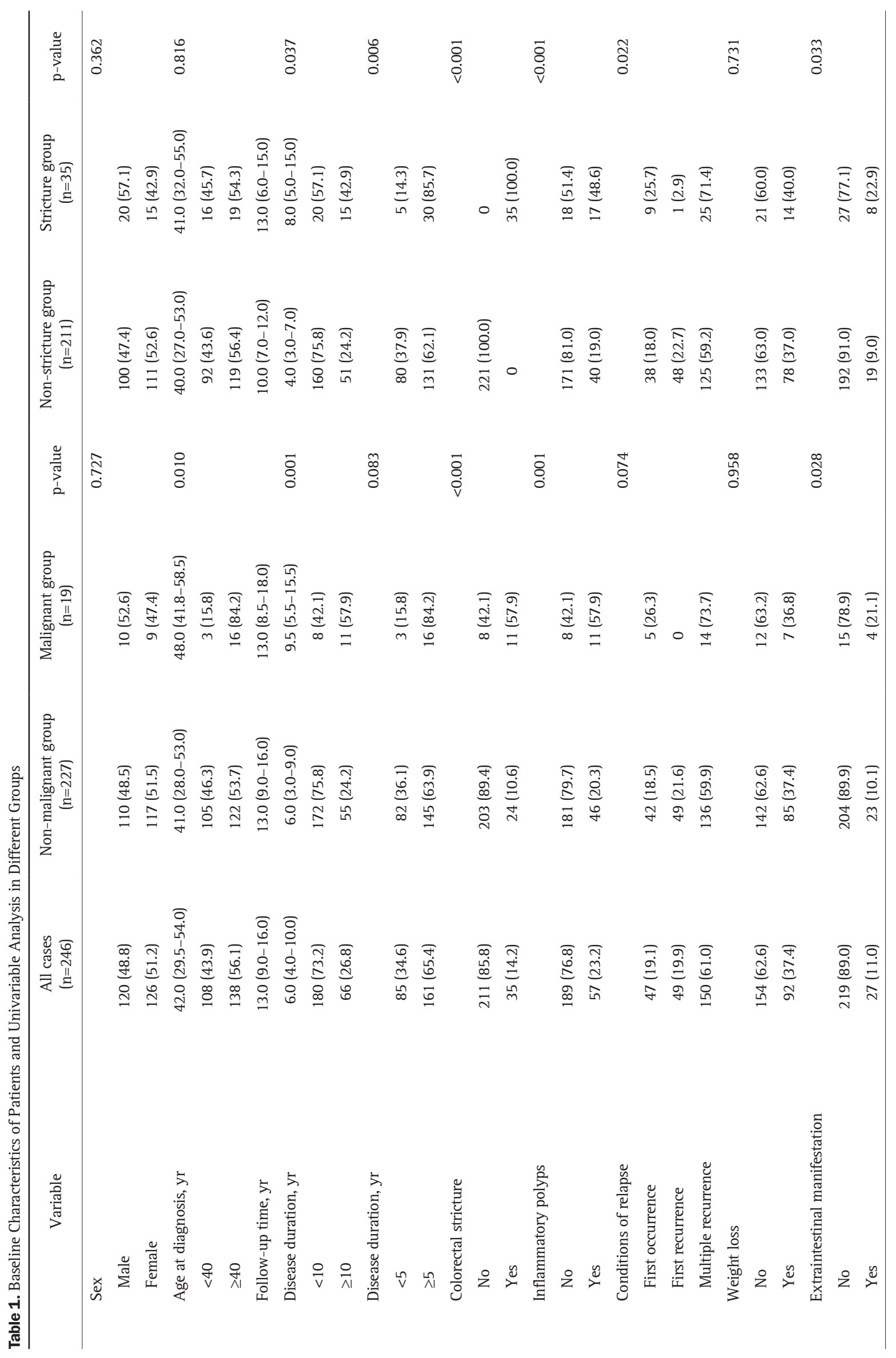




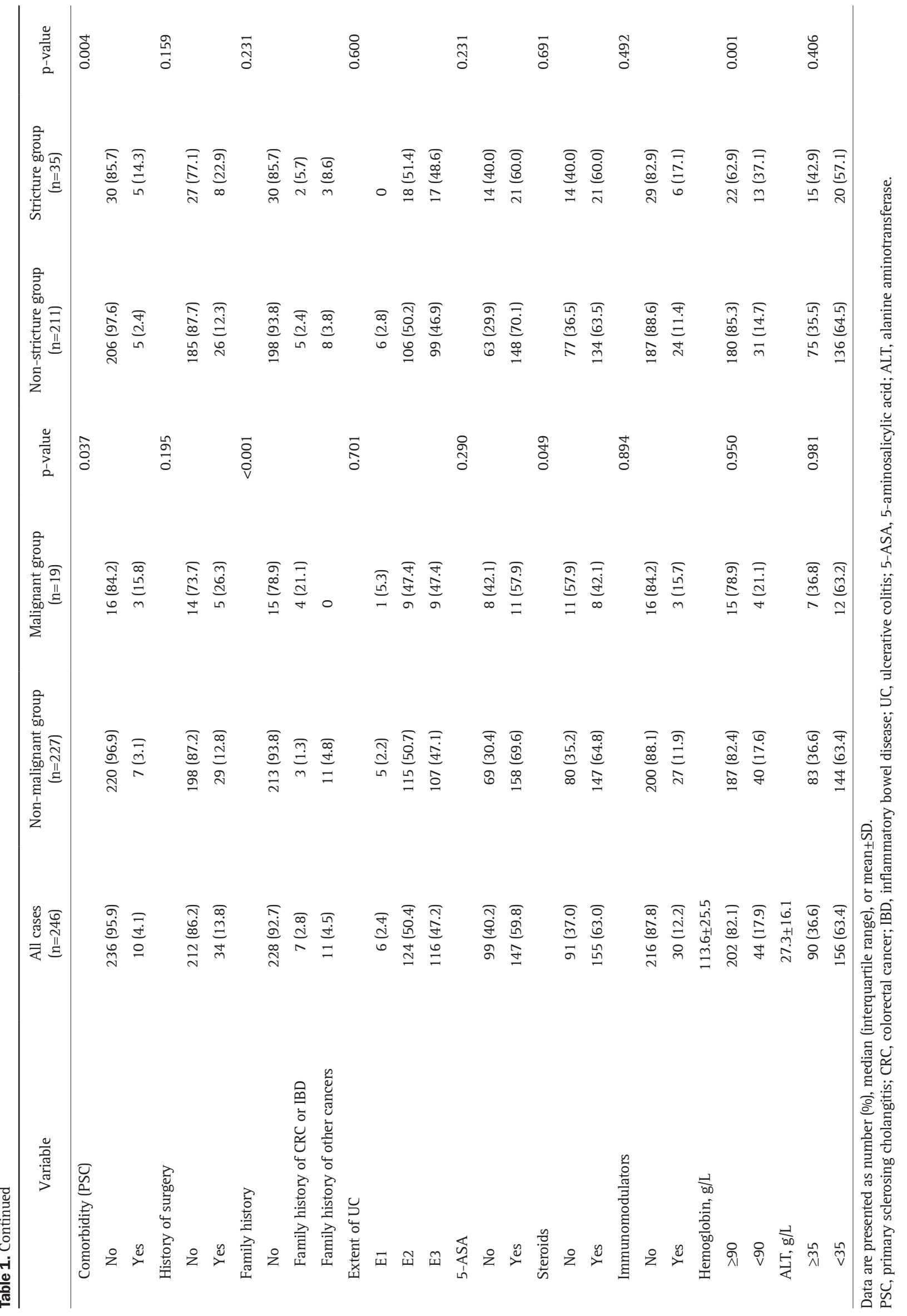




\section{Comparison of clinical outcomes between UC patients with and without stricture}

To identify whether colorectal stricture was a risk factor for poor prognosis in UC, we then compared the outcomes between patients with and without stricture (Table 3). After regular medical treatment, remission was more commonly seen in the nonstricture group ( $\mathrm{n}=109,51.7 \%)$ than that in the stricture group $(n=9,25.7 \%)(p=0.006)$. The patients with stricture experienced serious complications (bleeding, intestinal obstruction, or per-

Table 2. Complications

\begin{tabular}{lc}
\hline Complications & No. (\%) \\
\hline Colorectal stricture & $35(14.2)$ \\
Inflammatory polyps & $57(23.2)$ \\
Intestinal obstruction & $21(8.5)$ \\
Colon perforation & $6(2.4)$ \\
Toxic megacolon & $2(0.8)$ \\
Crohn's disease & $2(0.8)$ \\
Abscess formation & $3(1.2)$ \\
High-grade dysplasia & $8(3.3)$ \\
Colorectal cancer & $11(4.5)$ \\
\hline
\end{tabular}

foration), and the frequency of surgery was higher than that in the non-stricture group (45.7\% vs $12.3 \%, \mathrm{p}<0.001$ and $71.4 \%$ vs $36.5 \%, \mathrm{p}<0.001)$. In addition, the number of patients who developed dysplasia ( $\mathrm{n}=2,5.7 \%)$ or CRC $(\mathrm{n}=9,25.7 \%)$ was higher in the stricture group than that in the non-stricture group $(n=11$, $31.4 \%$ and $n=8,3.8 \% ; p<0.001)$. Meanwhile, there were no statistical differences in the cure, disease activity status, and death rates $(\mathrm{p}>0.05)$.

\section{Risk factors for developing high-grade dysplasia or CRC in UC}

Previous studies indicated that colorectal stricture caused poor clinical outcomes and even increased the risk of malignant transformation. Thus, we further analyzed the risk factors associated with malignant transformation. Among the 246 patients, 11 developed CRC and eight developed high-grade dysplasia. In univariate analysis, age at diagnosis $(p=0.010)$, disease $d u-$ ration $(\mathrm{p}=0.001)$, colorectal stricture $(\mathrm{p}<0.001)$, inflammatory polyps $(\mathrm{p}=0.001)$, extraintestinal manifestation $(\mathrm{p}=0.028)$, PSC ( $p=0.037)$, and familial history of IBD or CRC $(p<0.001)$ were significantly associated with malignant transformation. No statistical differences in sex, relapse, weight loss, history of surgery, disease extent, use of steroids and immunomodulators, and general conditions were observed between the malignant

Table 3. Comparison of Clinical Outcomes between Ulcerative Colitis Patients with and without a Stricture

\begin{tabular}{|c|c|c|c|}
\hline Variable & Non-stricture group & Stricture group & $\mathrm{p}$-value \\
\hline Cure & & & 0.084 \\
\hline No & $193(91.5)$ & $35(100)$ & \\
\hline Yes & $18(8.5)$ & 0 & \\
\hline Remission & & & 0.006 \\
\hline No & $102(48.3)$ & $26(74.3)$ & \\
\hline Yes & $109(51.7)$ & $9(25.7)$ & \\
\hline Alternate or continuous disease activity & & & 1.000 \\
\hline No & $147(69.7)$ & $25(71.4)$ & \\
\hline Yes & $64(30.3)$ & $10(28.6)$ & \\
\hline Serious complications & & & $<0.001$ \\
\hline No & $185(87.7)$ & $19(54.3)$ & \\
\hline Yes & $26(12.3)$ & $16(45.7)$ & \\
\hline Surgery & & & $<0.001$ \\
\hline No & $134(63.5)$ & $10(28.6)$ & \\
\hline Yes & 77 (36.5) & $25(71.4)$ & \\
\hline Malignant transformation & & & $<0.001$ \\
\hline No & 203 (96.2) & $24(68.6)$ & \\
\hline Yes & $8(3.8)$ & $11(31.4)$ & \\
\hline Death & & & 0.156 \\
\hline No & 204 (96.7) & 32 (91.4) & \\
\hline Yes & 7 (3.3) & 3 (8.6) & \\
\hline
\end{tabular}

Data are presented as number (\%). 
Table 4. Multivariate Logistic Regression Analysis of Risk Factors for High-Grade Dysplasia or CRC in UC

\begin{tabular}{|c|c|c|c|c|}
\hline \multirow{2}{*}{ Variable } & \multicolumn{2}{|c|}{ Univariate } & \multicolumn{2}{|c|}{ Multivariate } \\
\hline & OR $(95 \% \mathrm{CI})$ & p-value & OR $(95 \% \mathrm{CI})$ & p-value \\
\hline Colorectal stricture & $11.630(4.261-31.747)$ & $<0.001$ & $9.350(2.842-30.762)$ & $<0.001$ \\
\hline Inflammatory polyps & $5.410(2.058-14.222)$ & 0.001 & $5.463(1.692-17.638)$ & 0.005 \\
\hline Age at diagnosis ( $\geq 40 \mathrm{yr}$ ) & 4.590 (1.301-16.189) & 0.018 & $8.499(1.903-37.956)$ & 0.005 \\
\hline PSC & $5.893(1.390-24.986)$ & 0.016 & $1.441(0.221-9.393)$ & 0.703 \\
\hline Family history (CRC or IBD) & $1.488(0.646-3.422)$ & 0.349 & - & - \\
\hline Steroids & $0.396(0.153-1.024)$ & 0.056 & - & - \\
\hline
\end{tabular}

CRC, colorectal cancer; UC, ulcerative colitis; OR, odds ratio; CI, confidence interval; PSC, primary sclerosing cholangitis; IBD, inflammatory bowel disease.

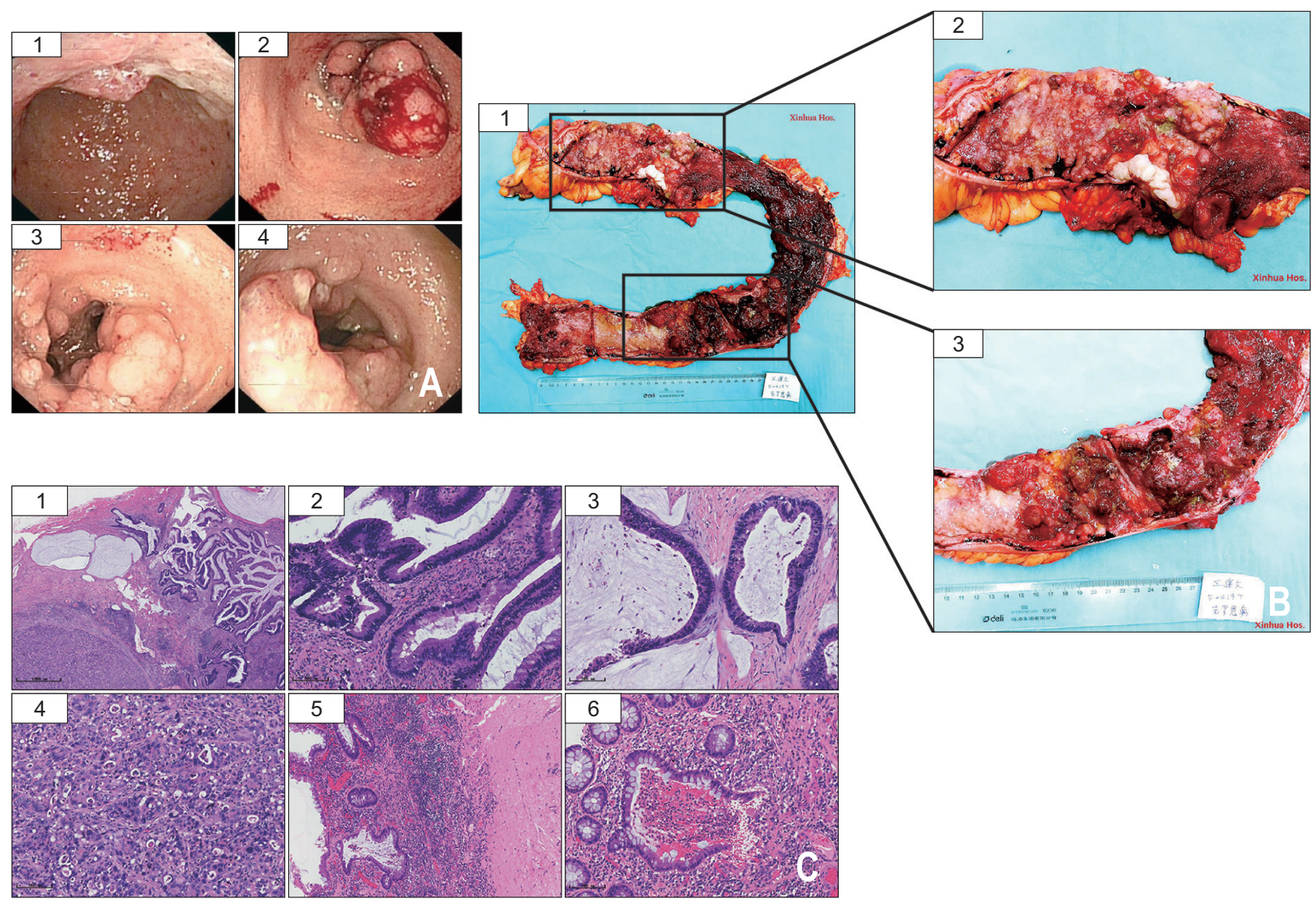

Fig. 1. Histopathological changes, endoscopic view, and gross resected lesion in the colon in an ulcerative colitis patient. (A) Colonic inflammation (A1), a serious stricture (A2), and dysplasia (A3 and A4) were detected in the endoscopic view. (B) The gross surgical specimen of the lesion in the colon (B1); dysplasia and adenocarcinoma (B2) and a severe colorectal stricture (B3) were observed. (C) Different grades of dysplasia and adenocarcinomas were observed in the same slice of the ascending colon (C1, H\&E, $\times 12.5)$, including tubulovillous adenoma (C2, H\&E, $\times 100)$, mucinous adenocarcinoma $(\mathrm{C} 3, \mathrm{H} \& \mathrm{E}, \times 100)$, and poorly differentiated adenocarcinoma (C4, $\mathrm{H} \& \mathrm{E}, \times 100)$. Multiple intestinal inflammatory changes were noted, and these mainly presented as edema and fibrosis (C5, H\&E, ×50) and a large crypt abscess (C6, H\&E, ×100).

group and non-malignant groups (Table 1). Table 4 shows a multivariable logistic regression model for the description of risk factors associated with malignant transformation in UC patients. In multivariate logistic analysis, colorectal stricture (OR, 9.350; 95\% CI, 2.842 to 30.762), inflammatory polyps (OR, 5.463; 95\%
CI, 1.692 to 17.638), age more than 40 years at diagnosis (OR, 8.499; 95\% CI, 1.903 to 37.956), and disease duration more than 10 years (OR, 3.223; 95\% CI, 1.040 to 9.985) were risk factors for developing high-grade dysplasia or CRC.

To further show the carcinogenic risk of stricture, we present 
Table 5. Multivariate Logistic Regression Analysis of Risk Factors for Colorectal Stricture Formation in UC

\begin{tabular}{|c|c|c|c|c|}
\hline \multirow{2}{*}{ Variable } & \multicolumn{2}{|c|}{ Univariate } & \multicolumn{2}{|c|}{ Multivariate } \\
\hline & OR $(95 \% \mathrm{CI})$ & p-value & OR $(95 \% \mathrm{CI})$ & p-value \\
\hline Disease duration ( $\geq 5 \mathrm{yr}$ ) & 3.664 (1.366-9.829) & 0.010 & $3.221(1.168-8.881)$ & 0.024 \\
\hline Moderate anemia & $3.431(1.566-7.518)$ & 0.002 & $3.373(1.472-7.731)$ & 0.004 \\
\hline PSC & $6.687(1.876-25.131)$ & 0.004 & $5.842(1.395-24.468)$ & 0.016 \\
\hline Multiple recurrence & 1.076 (0.678-1.706) & 0.756 & - & - \\
\hline
\end{tabular}

UC, ulcerative colitis; OR, odds ratio; CI, confidence interval; PSC, primary sclerosing cholangitis.

here the endoscopic view (Fig. 1A) of a grossly resected lesion in the colon with stricture and dysplasia (Fig. 1B) and the histopathological change (Fig. 1C) in a UC patient with longterm disease duration.

\section{Risk factors for colorectal stricture in UC patients}

Based on the above results, UC patients who developed colorectal stricture had the highest OR to develop malignant transformation. Therefore, we then analyzed the certain factors that contributed to the development colorectal stricture. In univariable analysis, disease duration more than 5 years $(p=0.006)$, multiple relapse $(p=0.022)$, extraintestinal manifestation ( $p=0.033)$, comorbidity (PSC) $(p=0.004)$, and moderate anemia (hemoglobin $<90 \mathrm{~g} / \mathrm{L})(\mathrm{p}=0.001)$ were significantly associated with colorectal stricture formation. There was no difference in sex, age at diagnosis, weight loss, history of surgery, familial history of IBD or CRC, disease extent, use of steroids and immunomodulators, and the level of alanine aminotransferase (Table 1). In multivariate logistic analysis, disease duration more than 5 years (OR, 3.211; 95\% CI, 1.168 to 8.881), moderated anemia (OR, 3.373; 95\% CI, 1.472 to 7.731), and PSC (OR, 5,842; 95\% CI, 1.395 to 24.468$)$ contributed to the development of colorectal stricture (Table 5).

\section{Analysis in 11 UC patents developed colorectal stricture and CRC}

To further evaluate the relationship between site of the stricture and malignant colonic segment, we performed analysis in 11 UC patients developed colorectal stricture and CRC. Theses 11 UC patients had colorectal stricture and developed malignant transformation. The number of strictures in each colorectal segment in 35 stricture patients (including multi-segmental stricture) is as follows: seven strictures were in ascending colon, five in transverse colon, 10 in descending colon, 11 in sigmoid colon and five in rectum, respectively. Then we found that two ascending colon strictures, three transverse colon strictures, three descending colon strictures, five sigmoid colon strictures, and one rectal stricture developed malignant lesions. In 11 cases, two malignant lesions were diagnosed in ascending colon, three in transverse colon, three in descending colon, five in sigmoid colon, and one in rectum. Therefore, we found that sigmoid colon was most prone to develop stricture (28.2 \%) and sigmoid colon stricture had the highest risk to develop malignant lesion (12.8\%). In the site of CRC, sigmoid colon malignancy was the most common (35.7\%). Additionally, seven patients (63.6\%) developed malignant lesion at the proximal location to the primary stricture, which further indicated that proximal bowel segment to the stricture was prone to develop malignant transformation.

\section{DISCUSSION}

Colorectal stricture remains a serious and intractable complication in UC and is a treatment challenge for clinicians. Patients with colorectal stricture more frequently have a poor clinical outcome, and even develop high-grade dysplasia and CRC. Although previous research already showed that stricture was associated with malignant transformation, few studies have investigated the risk factors of the stricture. We conducted herein the largest multicenter study in China evaluating the risk of dysplasia or CRC and colorectal stricture in UC patients. The major findings that we discovered in this study can be summarized as follows: (1) UC patients with colorectal stricture had worse clinical outcomes, and these included lower remission rates, more complications, need for surgery, and even malignant transformation; (2) colorectal stricture, inflammatory polyps, higher age at diagnosis, and long-term disease duration were significantly associated with dysplasia/CRC; (3) disease duration more than 5 years, moderate anemia, and the presence of PSC were risk factors for colorectal stricture in UC.

Although colitis-associated neoplasia constitutes only $1 \%$ to $2 \%$ of all colorectal carcinomas, ${ }^{8}$ it is the most serious complications for UC patients. Several studies have reported that UC patients with colonic stricture have a $0 \%$ to $33 \%$ risk of developing CRC. ${ }^{11,12,17,18}$ In our study, colorectal stricture was confirmed as the most important risk factor for malignant transformation (OR, 9.350; 95\% CI, 2.842 to 30.762; p<0.001), decreased remission rate, and increased incidence of colectomy and complications. Among the 246 patients, 35 patients (14.2\%) developed colorectal strictures. Of them, nine patients (25.7\%) were diag- 
nosed with CRC and two patients (5.7\%) developed high-grade dysplasia. The incidence of dysplasia or CRC in UC patients with stricture was similar to those in previous studies. Moreover, we found that inflammatory polyps, which are detected on colonoscopy, also increased the risk of dysplasia. UC-associated carcinogenesis is a complex process and can be summarized as a sequence of "inflammation-dysplasia-carcinoma," showing that the intestine exposed to chronic inflammation is at higher risk of developing dysplasia. ${ }^{19}$ Long-term chronic intestinal inflammation with recurrent relapse could be the cause for the formation of inflammatory polyps, which may accelerate the process of the above sequence, to promote malignant transformation.

To detect precancerous dysplasia or asymptomatic cancer, colonoscopic surveillance, as recommended by the American or British guidelines, ${ }^{20,21}$ was performed in UC patients. However, Lutgens et $a .^{22}$ reported that the diagnosis of CRC was delayed or missed in almost approximately $17 \%$ to $25 \%$ of patients after compliance with the recommended guidelines. In addition, definite diagnose of dysplasia or CRC via endoscopic biopsies is challenging, and negative biopsies cannot completely rule out the possibility of malignant transformation. ${ }^{23}$ Therefore, predictive factors of UC-associated carcinogenesis need to be identified, and the initial strategy of colonoscopic surveillance should be adjusted.

As reported previously, patients with colorectal stricture have the highest risk of developing dysplasia, but few studies have investigated the causative factors of stricture. Notably, we first discovered in this study that disease duration, moderate anemia, and the presence of PSC were significantly associated with stricture. These findings could have crucial implications in the timely identification of individuals at risk for stricture and thus allow earlier colonoscopic surveillance for detecting dysplasia at the beginning of UC.

Anemia is a common clinical manifestation in UC patients. A recent meta-analysis on the prevalence of anemia in IBD in Europe reported that anemia occurred in $24 \%$ of IBD patients, in $27 \%$ of $\mathrm{CD}$, and $21 \%$ in $\mathrm{UC},{ }^{24}$ respectively. In our study, $17.9 \%$ of patients experienced moderate anemia, which was lower than the reported values of $21 \%$. To identify a more sensitive risk factor, we specified anemia to moderate anemia at a hemoglobin value $<90 \mathrm{~g} / \mathrm{L}$. We believed that the value lower than the more common $<110 \mathrm{~g} / \mathrm{L}$ would result in lower frequency of anemia. Vegh et al. ${ }^{25}$ reported that anemia was associated with the transformation from non-stricture to stricture/penetrating and also increased the hospitalization rate in both UC and CD. In addition, research indicated that vitamin $\mathrm{B}_{12}$ deficiency was associated with higher rate of complications in CD patients, particularly stricture ( $p=0.013$ ) and the need for intestinal surgery $(\mathrm{p}=0.002)$. Further, CD patients with iron-deficiency anemia are at higher risk of developing intestinal stricture $(\mathrm{p}=0.001)$ and fistula ( $\mathrm{p}=0.004){ }^{26}$ The Crohn's Disease Activity Index was higher in patients with anemia, indicating that they underwent higher disease severity. This can be explained that by the fact that in general, anemia leads to long-term intestinal and extraintestinal complications. ${ }^{26}$ Moreover, a previous study reported that higher disease activity increased the risk of anemia. ${ }^{27}$ Therefore, UC patients with anemia usually develop chronic and serious inflammatory response, which ultimately leads to complications and poor outcome.

Another contributing factor for colorectal stricture is PSC, which is a chronic inflammatory disease of the biliary tree resulting in biliary cirrhosis, hepatic fibrosis, and increased risk of cholangiocarcinoma. ${ }^{28,29}$ Previous research indicated that patients with UC and concomitant PSC had higher risk of developing dysplasia or CRC. ${ }^{30}$ However, in the present study, we found that PSC was not significantly associated with malignant transformation. Similar results were reported by Braden et al. ${ }^{31}$ and Navaneethan et $a .^{32}$ However, interestingly, we discovered that PSC can significantly increase the risk of developing colorectal stricture in UC. There were 10 UC patients (4.1\%) who also had PSC; of them, six (60\%) had colorectal stricture. The characteristic chronic bile duct inflammation in PSC is considered to arise from a complex interaction between immunological and environmental factors in a genetically predisposed individual; this then leads to biliary strictures and ultimately to cirrhosis. Recently, Lampinen et $a{ }^{33}$ reported that serum soluble CD40 was significantly upregulated in UC patients with PSC. Donaldson et al. ${ }^{34}$ reported that the soluble CD40 ligand in proximal areas of stenosis of the superficial femoral artery increased immediately. Similarly, previous literatures also indicated that CD40/CD40 ligand is associated with inflammation in atherothrombosis ${ }^{35}$ and contributed to the development of renal artery stenosis. ${ }^{36}$ Interestingly, Friedrich et al. ${ }^{37}$ discovered that elevated serum soluble CD41 levels are beneficial in prevent the formation of biliary stricture in PSC patients. Collectively, these findings indicate that complex innate immune response could be involved in the development of UCPSC, including regulated CD14 and CD40 signaling. This could explain why PSC increased the risk of colorectal stricture in UC patients.

Colonoscopic surveillance guidelines recommended that surveillance should be initiated after 8 to 10 years of extensive colitis or after 15 to 20 years for left-sided colitis. However, the findings of the present study show that following such timing may delay or cause missed diagnosis of dysplasia in UC patients. Therefore, clinicians should pay more attention on the risk of colorectal stricture formation in UC patients with moderate anemia or concomitant PSC and disease duration more than 5 years. Appropriate and earlier endoscopic biopsies should be conducted when colorectal stricture occurs, particularly in patients with the above risk factors for stricture formation.

It is important to identify the formation time of stricture and malignant lesion, while we found that no patients developed stricture and malignant lesion at the same time. These patients developed malignant transformation after a long disease dura- 
tion of 8.0 years (range, 6.0 to 13.0 years). Strictures in patients with UC are rare but may harbor or be the result of malignancy. The pathogenesis of stricture formation in UC remains uncertain. It is not certain whether strictures degenerate into malignancy or whether malignant strictures are carcinomas to start with. It is difficult on the basis of the present retrospective analysis to make definitive recommendations for the management of strictures. This study at least indicated that colorectal stricture formation should be taken into full consideration in clinical practice.

There are limitations to our study. First, there could have been some selection bias because all included patients were diagnosed and managed by physicians from two different departments (colorectal surgeons and gastroenterology clinicians). Second, due to the retrospective nature and long duration of this study, loss to follow-up and incomplete clinical data are inevitable.

Colorectal stricture, inflammatory polyps, age $>40$ years at diagnosis, and 10-year disease duration was associated with malignant transformation in UC. Of these factors, colorectal stricture has the highest risk of causing high-grade dysplasia or CRC. UC patients with disease duration more than 5 years, moderate anemia, or PSC should be closely monitored via colonoscopic surveillance for the occurrence of colorectal stricture and further malignant transformation. Earlier initiation of colonoscopic surveillance in UC patients with risk factors for stricture is recommended for timely diagnosis of precancerous dysplasia or asymptomatic cancer and for more appropriate and individualized treatment.

\section{CONFLICTS OF INTEREST}

No potential conflict of interest relevant to this article was reported.

\section{ACKNOWLEDGEMENTS}

This work was supported by grants from the National Natural Science Foundation of China (81873547) and Medical and Engineering Crossing Foundation of Shanghai Jiaotong University (YG2017MS71).

\section{AUTHOR CONTRIBUTIONS}

Study design: Y.G., P.D. Data analysis and writing the manuscript: W.X. Statistics of the data in the revised manuscript and the re-examination of the previous data: W.D. Collaboration to collect the information of patients: L.C., J.Z. All authors participated in revising the manuscript and approved the final version.

\section{ORCID}

Weimin $\mathrm{Xu}$ Wenjun Ding https://orcid.org/0000-0002-6607-5086

https://orcid.org/0000-0002-9285-5318
Yubei Gu

https://orcid.org/0000-0002-4940-5303

Long Cui https://orcid.org/0000-0003-1838-4471

Jie Zhong https://orcid.org/0000-0003-4499-7263

Peng Du

https://orcid.org/0000-0001-9355-0789

\section{REFERENCES}

1. Kaplan GG, Ng SC. Understanding and preventing the global increase of inflammatory bowel disease. Gastroenterology 2017;152:313-321.

2. Melmed GY, Targan SR. Future biologic targets for IBD: potentials and pitfalls. Nat Rev Gastroenterol Hepatol 2010;7:110-117.

3. Marchioni Beery R, Kane S. Current approaches to the management of new-onset ulcerative colitis. Clin Exp Gastroenterol 2014;7:111-132.

4. Ording AG, Horváth-Puhó E, Erichsen R, et al. Five-year mortality in colorectal cancer patients with ulcerative colitis or Crohn's disease: a nationwide population-based cohort study. Inflamm Bowel Dis 2013;19:800-805.

5. Ekbom A, Helmick C, Zack M, Adami HO. Ulcerative colitis and colorectal cancer: a population-based study. N Engl J Med 1990;323:1228-1233.

6. Jess T, Rungoe C, Peyrin-Biroulet L. Risk of colorectal cancer in patients with ulcerative colitis: a meta-analysis of populationbased cohort studies. Clin Gastroenterol Hepatol 2012;10:639-645.

7. Derikx LA, Nissen LH, Smits LJ, Shen B, Hoentjen F. Risk of neoplasia after colectomy in patients with inflammatory bowel disease: a systematic review and meta-analysis. Clin Gastroenterol Hepatol 2016;14:798-806.

8. Eaden JA, Abrams KR, Mayberry JF. The risk of colorectal cancer in ulcerative colitis: a meta-analysis. Gut 2001;48:526-535.

9. Yamagata M, Mikami T, Tsuruta T, et al. Submucosal fibrosis and basic-fibroblast growth factor-positive neutrophils correlate with colonic stenosis in cases of ulcerative colitis. Digestion 2011;84:12-21.

10. Edwards FC, Truelove SC. The course and prognosis of ulcerative colitis: III. complications. Gut 1964;5:1-22.

11. De Dombal FT, Watts JM, Watkinson G, Goligher JC. Local complications of ulcerative colitis: stricture, pseudopolyposis, and carcinoma of colon and rectum. Br Med J 1966;1:1442-1447.

12. Gumaste V, Sachar DB, Greenstein AJ. Benign and malignant colorectal strictures in ulcerative colitis. Gut 1992;33:938-941.

13. Fumery M, Pineton de Chambrun G, Stefanescu C, et al. Detection of dysplasia or cancer in 3.5\% of patients with inflammatory bowel disease and colonic strictures. Clin Gastroenterol Hepatol 2015;13:1770-1775.

14. Silverberg MS, Satsangi J, Ahmad T, et al. Toward an integrated clinical, molecular and serological classification of inflammatory bowel disease: report of a Working Party of the 2005 Montreal World Congress of Gastroenterology. Can J Gastroenterol 2005;19 Suppl A:5A-36A.

15. Lee HW, Park SJ, Jeon SR, et al. Long-term outcomes of endo- 
scopic balloon dilation for benign strictures in patients with inflammatory bowel disease. Gut Liver 2018;12:530-536.

16. Xu W, Ye H, Zhu Y, et al. Long-term quality of life associated with early surgical complications in patients with ulcerative colitis after ileal pouch-anal anastomosis: a single-center retrospective study. Int J Surg 2017;48:174-179.

17. Lashner BA, Turner BC, Bostwick DG, Frank PH, Hanauer SB. Dysplasia and cancer complicating strictures in ulcerative colitis. Dig Dis Sci 1990;35:349-352.

18. Hunt RH, Teague RH, Swarbrick ET, Williams CB. Colonoscopy in management of colonic strictures. Br Med J 1975;3:360-361.

19. Itzkowitz SH, Yio X. Inflammation and cancer IV. Colorectal cancer in inflammatory bowel disease: the role of inflammation. Am J Physiol Gastrointest Liver Physiol 2004;287:G7-G17.

20. Winawer S, Fletcher R, Rex D, et al. Colorectal cancer screening and surveillance: clinical guidelines and rationale-update based on new evidence. Gastroenterology 2003;124:544-560.

21. Eaden JA, Mayberry JF; British Society for Gastroenterology; Association of Coloproctology for Great Britain and Ireland. Guidelines for screening and surveillance of asymptomatic colorectal cancer in patients with inflammatory bowel disease. Gut 2002;51 Suppl 5:V10-V12.

22. Lutgens MW, Vleggaar FP, Schipper ME, et al. High frequency of early colorectal cancer in inflammatory bowel disease. Gut 2008;57:1246-1251.

23. Rutter MD, Saunders BP, Wilkinson KH, et al. Cancer surveillance in longstanding ulcerative colitis: endoscopic appearances help predict cancer risk. Gut 2004;53:1813-1816.

24. Filmann N, Rey J, Schneeweiss S, et al. Prevalence of anemia in inflammatory bowel diseases in European countries: a systematic review and individual patient data meta-analysis. Inflamm Bowel Dis 2014;20:936-945.

25. Vegh Z, Kurti Z, Gonczi L, et al. Association of extraintestinal manifestations and anaemia with disease outcomes in patients with inflammatory bowel disease. Scand J Gastroenterol 2016;51:848-854.

26. Madanchi M, Fagagnini S, Fournier N, et al. The relevance of vitamin and iron deficiency in patients with inflammatory bowel diseases in patients of the Swiss IBD Cohort. Inflamm Bowel Dis 2018;24:1768-1779.

27. Bergamaschi G, Di Sabatino A, Albertini R, et al. Prevalence and pathogenesis of anemia in inflammatory bowel disease: influence of anti-tumor necrosis factor-alpha treatment. Haematologica 2010;95:199-205.

28. Wiesner RH, LaRusso NF. Clinicopathologic features of the syndrome of primary sclerosing cholangitis. Gastroenterology 1980;79:200-206.

29. Rosen CB, Nagorney DM. Cholangiocarcinoma complicating primary sclerosing cholangitis. Semin Liver Dis 1991;11:26-30.

30. Shetty K, Rybicki L, Brzezinski A, Carey WD, Lashner BA. The risk for cancer or dysplasia in ulcerative colitis patients with primary sclerosing cholangitis. Am J Gastroenterol 1999;94:1643-1649.

31. Braden B, Halliday J, Aryasingha S, et al. Risk for colorectal neoplasia in patients with colonic Crohn's disease and concomitant primary sclerosing cholangitis. Clin Gastroenterol Hepatol 2012;10:303-308.

32. Navaneethan U, Rai T, Venkatesh PG, Kiran RP. Primary sclerosing cholangitis and the risk of colon neoplasia in patients with Crohn's colitis. Gastroenterol Rep (Oxf) 2016;4:226-231.

33. Lampinen M, Vessby J, Fredricsson A, Wanders A, Rorsman F, Carlson M. High serum sCD40 and a distinct colonic T cell profile in ulcerative colitis associated with primary sclerosing cholangitis. J Crohns Colitis 2019;13:341-350.

34. Donaldson CW, Schneider DJ, Bertges DJ, et al. Increased local cytokine production at culprit superficial femoral artery plaques. J Thromb Thrombolysis 2013;36:293-299.

35. Antoniades C, Bakogiannis C, Tousoulis D, Antonopoulos AS, Stefanadis C. The CD40/CD40 ligand system: linking inflammation with atherothrombosis. J Am Coll Cardiol 2009;54:669-677.

36. Haller ST, Evans KL, Folt DA, Drummond CA, Cooper CJ. Mechanisms and treatments for renal artery stenosis. Discov Med 2013;16:255-260.

37. Friedrich K, Smit M, Brune M, et al. CD14 is associated with biliary stricture formation. Hepatology 2016;64:843-852. 